Research Article

\title{
Dioscin Attenuates Myocardial Ischemic/Reperfusion-Induced Cardiac Dysfunction through Suppression of Reactive Oxygen Species
}

\author{
Dayin Lyu, ${ }^{1}$ Qing Tian, ${ }^{2}$ Huitao Qian, ${ }^{3}$ Chang He, ${ }^{1}$ Tianyu Shen, ${ }^{4}$ Jinxing Xi, ${ }^{5}$ \\ Pingxi Xiao $\mathbb{1}^{6},{ }^{6}$ and Qiulun $L u \mathbb{B}^{1}$ \\ ${ }^{1}$ Key Laboratory of Cardiovascular and Cerebrovascular Medicine, Collaborative Innovation Center for Cardiovascular Disease \\ Translational Medicine, Nanjing Medical University, Nanjing 211166, China \\ ${ }^{2}$ Intensive Care Unit of Wuhan Asia Heart Hospital, Wuhan 430000, China \\ ${ }^{3}$ The First Clinical Medical College of Nanjing Medical University, Nanjing 211166, China \\ ${ }^{4}$ Department of Orthopedics Surgery, Taihe Hospital, Hubei University of Medicine, Shiyan 442000, China \\ ${ }^{5}$ Nanjing Vocational College of Information Technology, Nanjing 211166, China \\ ${ }^{6}$ Cardiac Department, Sir Runrun Hospital Affiliated to Nanjing Medical University, Nanjing 211166, China
}

Correspondence should be addressed to Pingxi Xiao; sysu-xiao@163.com and Qiulun Lu; qiulunlu@njmu.edu.cn

Dayin Lyu and Qing Tian contributed equally to this work.

Received 15 July 2021; Accepted 2 September 2021; Published 5 October 2021

Academic Editor: Ding-Sheng Jiang

Copyright (c) 2021 Dayin Lyu et al. This is an open access article distributed under the Creative Commons Attribution License, which permits unrestricted use, distribution, and reproduction in any medium, provided the original work is properly cited.

\begin{abstract}
Myocardial ischemic/reperfusion (MI/R) is a leading cause of cardiovascular disease with high morbidity and mortality. However, the mechanisms underlying pathological reperfusion remain obscure. In this study, we found that dioscin, a natural product, could be a potential candidate for treating MI/R through modulating cardiac dysfunction. Mechanistically, our work revealed that dioscin could suppress the production of reactive oxygen species (ROS) via repressing the nicotinamide adenine dinucleotide phosphate (NADPH) oxidase 2 (Nox2) and enhancing the expression of antioxidant enzymes, including superoxide dismutase (SOD), catalase (CAT), glutathione (GSH), and glutathione peroxidase (GPx). These findings indicate that dioscin may be a potential candidate for therapeutic interventions in MI/R injury.
\end{abstract}

\section{Introduction}

Myocardial ischemic/reperfusion (MI/R), a leading cause of cardiovascular disease with high morbidity and mortality, is caused by the blood recovery after a vital period of coronary artery occlusion, which easily leads to myocardial infarction or heart failure [1-3]. Previously, literature reported that lethal reperfusion injury accounts for up to $50 \%$ of the final myocardial infarct size [4]. It is well known that the pathogenesis of $\mathrm{MI} / \mathrm{R}$ injury includes oxidative stress, inflammatory response, calcium overload, and mitochondrial dysfunction [5-8]. Since the oxidant stress is accompanied with the pathological process of cardiac dysfunction, inhibition of oxidant stress is a potential therapeutic strategy for MI/R injury [9].

Oxidative stress is originated from the overwhelmed reactive oxygen species (ROS) and the insufficient antioxidant defense systems [10]. Under the physiological condition, ROS is crucial and maintains normal cellular metabolism processes, balancing at the dynamic stage between antioxidants and oxidant response [11]. However, abundant of ROS is generated in two stages of MI/R, including ischemia and reperfusion [12]. Due to sudden overburden of the high oxygen 
tensions, reperfusion contributes to elevated levels of oxygen free radical (OFR) production, which leads to oxidative damage, such as protein carbonylation and DNA oxidation $[13,14]$. Nevertheless, various traditional antioxidants do not present with therapeutic efficacy $[15,16]$. Some active natural products, including pentamethylquercetin, isorhychophylline, myricetin, and fisetin from medicinal plants, have shown excellent activities against MI/R injury [17-20]. Thus, it is reasonable to exploit effective natural products from herbs for the treatment of MI/R injury.

Dioscin, a natural steroid saponin isolated from the root bark of wild dioscorea nipponica, is currently widely used for cardiovascular disease treatment [21]. Our previous investigations have demonstrated that dioscin elevates lncRNA MANTIS in therapeutic angiogenesis for myocardial infarction [22]. What is more, dioscin plays a beneficial role in hepatic ischemia-reperfusion injury, intestinal ischemia-reperfusion injury, and gastric ischemia-reperfusion injury [23-25]. However, whether dioscin protects cardiac injury against MI/R remains unclear.

Hence, dioscin was systematically investigated in a MI/R-injured mouse model in vivo and in H9C2 cardiomyocytes in vitro subjected to hypoxia/reoxygenation (H/R) injury, presenting the cardioprotective role of dioscin and additionally demonstrating the beneficial function of dioscin against reactive oxygen species, in order to provide new options for the clinical treatment of MI/R.

\section{Materials and Methods}

2.1. Animals. Male C57BL/6 mice (22-24 g) were purchased from the Animal Core of Nanjing Medical University (Nanjing, China). The mice were kept with a standard vivarium with free access to food and water. All animal experiments were approved by the National Institutes of Health Guide for the Care and Use of Laboratory Animals, and the protocols used were also consistent with the Animal Ethics Committee of Nanjing Medical University, Nanjing, China (IACUC-2003006).

The myocardial ischemia/reperfusion injury model was produced as previously reported [26]. Briefly, the left anterior descending (LAD) coronary was tied by a slipknot with a 6-0 silk suture. After $30 \mathrm{~min}$ of ischemia, the slipknot was released smoothly and gently until a feeling of release was sensed, at which time, the myocardium began reperfusion and was kept for 3 days. Sham-operated control mice underwent the same surgical procedure, except the suture placed under the left coronary artery was not tied.

Dioscin (80 mg/kg/day, Di'ao Group, Chengdu, China) was administrated to mice for 3 days beginning on the day of operation. Briefly, the dioscin was first dissolved in dimethyl sulphoxide (DMSO), and then, 5\% sodium carboxymethyl cellulose (CMC-Na) was added to make the volume ratio for $1: 19$. Consequently, the mice were given the dissolution by intragastric administration. The preparation and application of the drug's dissolution should be completed in one day. If precipitation occurred in the preparation process, it could be assisted by heating or ultrasound.
2.2. Measurement of Cardiac Function by Echocardiography. Echocardiographic measurements were performed on mice using a VisualSonics Vevo ${ }^{\circledR} 2100$ Imaging System (VisualSonics, Toronto, Canada) with a $40 \mathrm{MHz}$ MicroScan transducer (model MS-550D) [27]. The M-mode echocardiogram was acquired from the parasternal short axis view of the left ventricle at the midpapillary muscle level. Echocardiographic parameters were calculated using the primary measurements and accompanying software. The echocardiographer was blinded to the genetic identity of the mice for all studies.

2.3. Histological Assays. Mouse hearts were dissected out, then fixed in 4\% paraformaldehyde (PFA, Electron Microscopy Sciences) overnight. After dehydration through a series of ethanol baths, samples were embedded in paraffin wax. Further, $5 \mu \mathrm{m}$ thick samples of the heart were obtained to perform hematoxylin and eosin (HE) and Masson trichrome staining. Slides were imaged under a light microscope.

2.4. Determination of Enzyme Activities. The detection kits of superoxide dismutase (SOD), catalase (CAT), glutathione (GSH), and glutathione peroxidase (GPx) were purchased from a company (Solarbio, China). The activities of these enzymes in the heart were evaluated following the manufacturer's instructions.

2.5. Determination of Reactive Oxygen Species (ROS). The production of ROS was measured as previously described [28]. The samples of the heart section were incubated with the DHE (Beyotime, China) at $37^{\circ} \mathrm{C}$ for $30 \mathrm{~min}$, then washed three times with PBS for $5 \mathrm{~min}$, and further costained with DAPI (Beyotime, China). The fluorescence intensity was examined using a confocal scanning microscope, and all images were analyzed using ImageJ software.

2.6. Quantitative RT-PCR. Total RNA was isolated using the TRIzol Reagent (Invitrogen) from cell or tissue samples. The mRNA expression levels were determined by quantitative reverse transcription polymerase chain reaction (PCR) using SuperScript II Reverse Transcriptase (Thermo Fisher Scientific Inc.) for reverse transcription and a Power SYBR Green PCR Master Mix (Thermo Fisher Scientific Inc.) for quantitative reverse transcription PCR reaction with PCR primers. The measurable of corresponding genes were detected by realtime PCR Detection System (Bio-Rad) and were analysed by CFX Manager 3.1 software (Bio-Rad). The sequences of primers were as follows: Anp-F: $5^{\prime}$-ACC TCC CGA AGC TAC CTA AGT-3', Anp-R: 3' -CAA CCT TTT CAA CGG CTC CAA-5'; Bnp-F: $5^{\prime}$-GAG GTC ACT CCT ATC CTC TGG-3', Bnp-R: $3^{\prime}$-GCC ATT TCC TCC GAC TTT TCT C- $5^{\prime}$; $\beta$-Mhc-F: $5^{\prime}$-GAG GGT GGC TCT CAC ACA TTC$3^{\prime}, \beta$-Mhc-R: $3^{\prime}$-TTG GCC TTC GTA AGC AAA CTG-5'; Sod1-F: $5^{\prime}$-AAC CAG TTG TGT TGT CAG GAC-3', Sod1R: $3^{\prime}$-CCA CCA TGT TTC TTA GAG TGA GG-5'; Sod2-F: 5'-CAG ACC TGC CTT ACG ACT ATG G-3', Sod2-R: 3' CTC GGT GGC GTT GAG ATT GTT-5'; Cat-F: $5^{\prime}$-AGC GAC CAG ATG AAG CAG TG-3', Cat-R: $3^{\prime}$-TCC GCT CTC TGT CAA AGT GTG-5'; Nrf2-F: $5^{\prime}$-CCA TTT ACG 


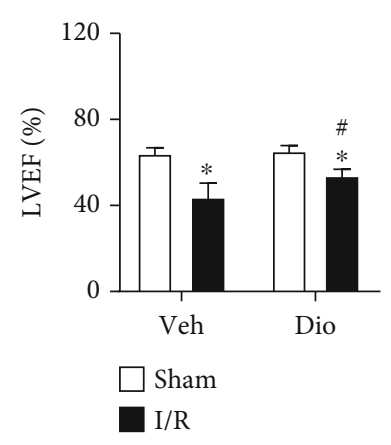

(a)

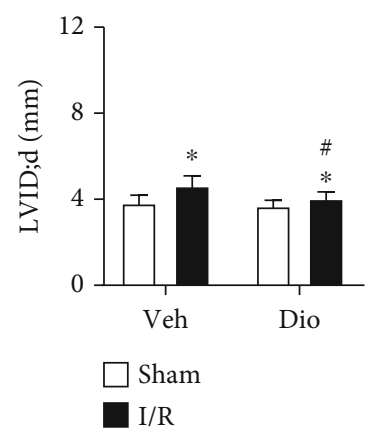

(c)

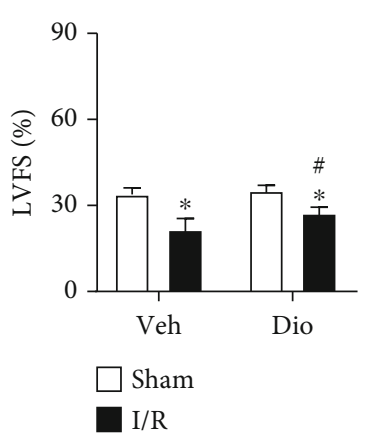

(b)

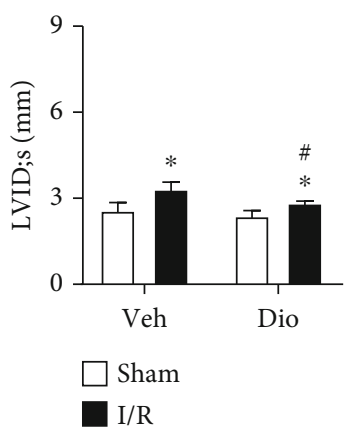

(d)

Figure 1: Dioscin improves cardiac function in ischemic/reperfusion mice. The C57BL/6 mice were subjected to myocardial ischemic/reperfusion (MI/R) surgery. Echocardiographic parameters for (a) left ventricular ejection fraction (LVEF, \%), (b) left ventricular fractional shortening (LVFS, \%), (c) left ventricular end diastolic internal dimension (LVID; $\mathrm{d}, \mathrm{mm}$ ), and (d) left ventricular end systolic internal dimension (LVID; s, mm). $n=8$ each group. Data are mean \pm SD. ${ }^{*} P<0.05$ vs. sham group, ${ }^{\#} P<0.05$ vs. I/R group.

GAG ACC CAC CGC CTG-3', Nrf2-R: $3^{\prime}$-CTC GTG TGA GAT GAG CCT CTA AGC GG-5'; Nox2-F: $5^{\prime}$-ACT CCT TGG GTC AGC ACT GG-3', Nox2-R: $3^{\prime}$-GTT CCT GTC CAG TTG TCT TCG-5'; and 18s-F: $5^{\prime}$-GCC TCC TCC TCC TCT CTC-3', 18s-R: $3^{\prime}$-GCT ACT GGC AGG ATC AAC C-5

2.7. Statistical Analysis. Continuous variables that approximated the normal distribution were expressed as mean \pm $\mathrm{SD}$. Comparison between groups was subjected to ANOVA followed by the Bonferroni correction for post hoc $t$-test. Data expressed as proportions were assessed with a chisquare test. Two-sided tests have been used throughout, and $P$ values $<0.05$ were considered statistically significant. GraphPad Prism 8 was used to evaluate data.

\section{Results}

3.1. Dioscin Improves Cardiac Dysfunction in MI/R-Injured Mice. In order to explore the protective role of dioscin in response to $\mathrm{MI} / \mathrm{R}$ injury in vivo, mice were subjected to myocardial ischemic/reperfusion surgery. Echocardiography exhibited that the significant increases of cardiac function markers of the left ventricular ejection fraction (LVEF) and the left ventricular fractional shortening (LVFS) were observed in the MI/R with the dioscin treatment group compared to the MI/R group (Figures $1(\mathrm{a})$ and $1(\mathrm{~b})$ ). Further- more, we found that mice subjected to MI/R treated with dioscin presented improved cardiac function, as evidenced by the preserved left ventricular end diastolic internal dimension (LVID; d) and left ventricular end systolic internal dimension (LVID; s), when compared with vehicle-treated mice (Figures 1(c) and 1(d)).

To examine whether the cardiac fibrosis was prevented by dioscin or not, a series of staining were performed on heart sections. Hematoxylin and eosin (HE) staining revealed widespread myocardial structural disorder, while treatment with dioscin markedly ameliorated histological features in myocardial tissue (Figure 2(a)). Moreover, collagen accumulation in the interstitial space, which was detected by Masson's trichrome staining, increased obviously in the heart sections of the MI/R group, and this increase was improved significantly in the MI/R with treatment dioscin group (Figures 2(b) and 2(c)). We further examined the expression of biomarkers for cardiac function, finding that the expression of natriuretic peptide $\mathrm{A}(A n p)$, natriuretic peptide $\mathrm{B}(B n p)$, and betamyosin heavy polypeptide cardiac muscle $(\beta-M h c)$ was reduced after being treated with dioscin in MI/R injury hearts compared to the MI/R group (Figures $2(\mathrm{~d})-2(\mathrm{f})$ ). These results indicated that dioscin improves cardiac function and alleviates cardiac fibrosis against MI/R injury.

3.2. Dioscin Modulates Antioxidant Status in MI/R Mice. Because of the involvement of ROS in MI/R injury, we detected the ROS levels in perfused hearts. Dihydroethidium 


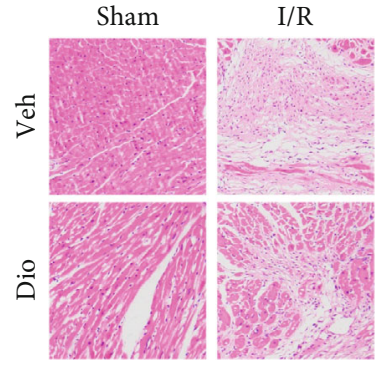

(a)

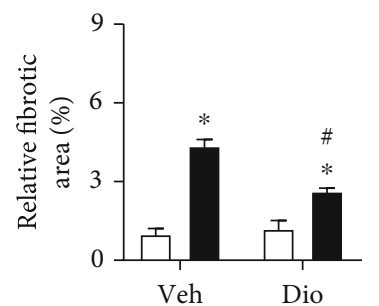

$\square$ Sham
$\square$ I/R

(c)

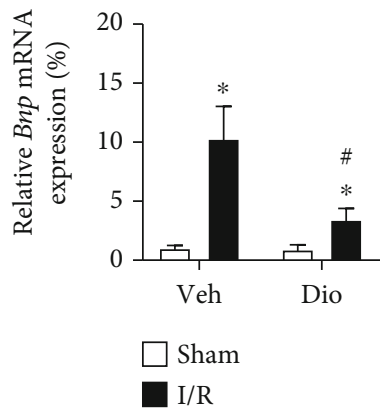

(e)

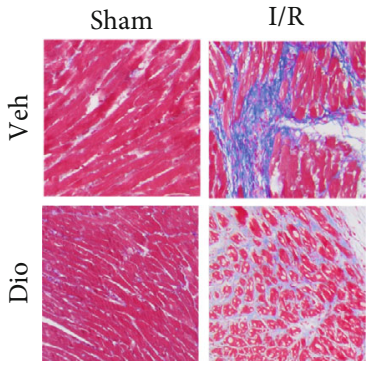

(b)

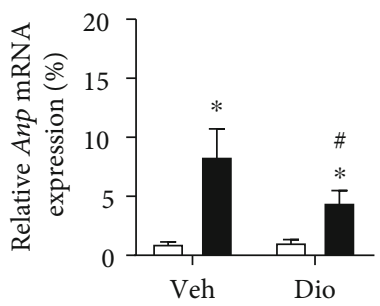

$\square$ Sham

$\mathrm{I} / \mathrm{R}$

(d)

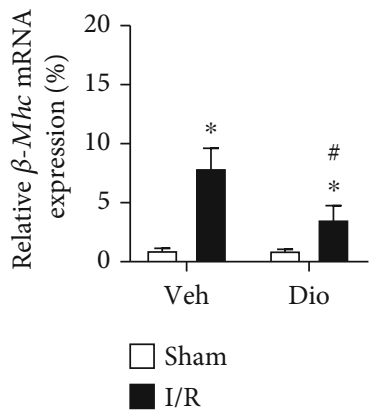

(f)

FiguRE 2: Dioscin alleviates cardiac fibrosis in MI/R injury mice. (a) Representative images of HE staining (bar $=100 \mu \mathrm{m}$ ), $n=3$ each group. (b) Representative images of Masson trichrome-stained ( $\mathrm{bar}=100 \mu \mathrm{m}), n=3$ each group. (c) The measurement of relative fibrosis area (\%). The mRNA expression of (d) Anp, (e) Bnp, and (f) $\beta$-Mhc. $n=6$ each group. Data are mean $\pm \mathrm{SD} .{ }^{*} P<0.05$ vs. sham group, ${ }^{\#} P<0.05$ vs. I/R group.

(DHE) staining was performed using heart sections, indicating that the levels of ROS in MI/R-induced heart tissues were significantly increased compared with those in the sham group, and dioscin treatment markedly attenuated the elevated production of ROS (Figures 3(a) and 3(b)).

To identify the mechanism underlying how dioscin regulates antioxidant stress in response to MI/R injury, we subsequently detected the mRNA level of corresponding oxidative genes. We found that the expressions of superoxide dismutase 1 (Sod1), superoxide dismutase 2 (Sod2), catalase (Cat), and nuclear factor erythroid 2-related factor 2 (Nrf2) were upregulated in hearts from the MI/R group compared to sham mice, which was prevented by dioscin treatment (Figures 3(c)-3(f)). These results might reveal that the upregulated expression of ROS in the period of perfusion causes the increase expression of antioxidant genes, while the production of ROS is eliminated by treatment with dioscin to further suppress the expression of antioxidant genes.
To further explore the mechanism underlying the protective role of dioscin against MI/R injury, we further measured the activities of antioxidant status-related enzymes. Superoxide dismutase (SOD) is the typical antioxidant enzyme as ROS scavenger. In the MI/R group, the activities of SOD were significant decreased compared to those of sham mice, but the downregulation was prevented by dioscin (Figure 4(a)). What is more, neither catalase (CAT), glutathione $(\mathrm{GSH})$, nor glutathione peroxidase (GPx) plays a protective role in cells from oxidative damage. Dioscin suppressed the declination of MI/R-induced activities of CAT, GSH, and GPx (Figures 4(b)-4(d)). We suspect that the dynamic balance between the antioxidants and oxidant response was damaged by an overburdened high ROS, which further injures these antioxidant enzymes. However, treatment with dioscin recues the decline. Taken together, dioscin could regulate the antioxidant status to repress the production of the ROS level in the MI/R heart. 


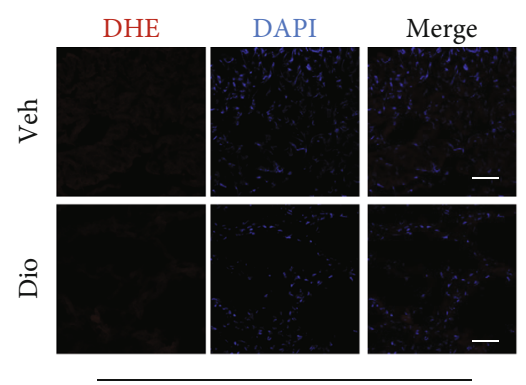

Sham

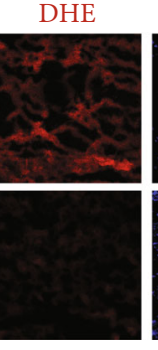

$\mathrm{I} / \mathrm{R}$

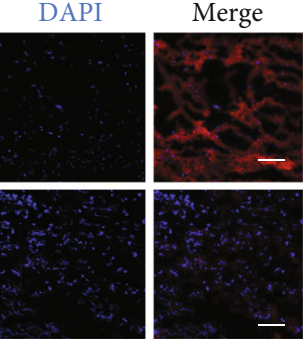

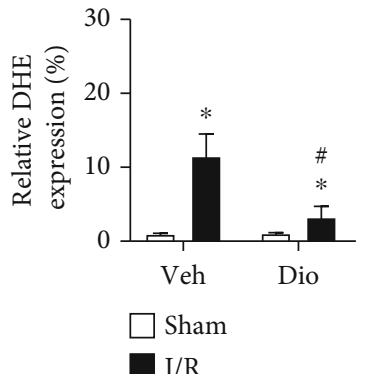

$\mathrm{I} / \mathrm{R}$

(b)

(a)

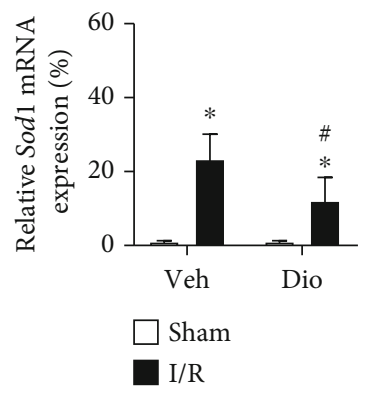

(c)

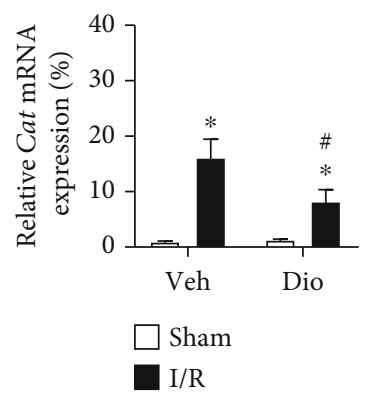

(e)

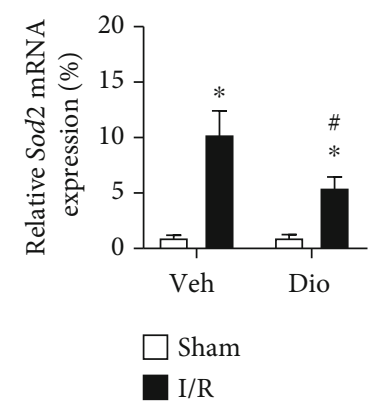

(d)

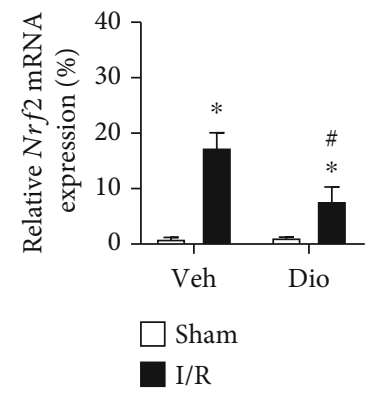

(f)

FiguRE 3: Dioscin changes the oxidant response after I/R. (a) Representative images of double staining of heart sections with dihydroethidium (DHE) (red) and DAPI (blue) (bar $=20 \mu \mathrm{m}$ ), $n=3$ each group. (b) The quantification of relative expression of reactive oxygen species. The mRNA expression of antioxidant genes, including (c) Sod1, (d) Sod2, (e) Cat, and (f) Nrf2. $n=6$ each group. Data are mean $\pm \mathrm{SD} .{ }^{*} P<0.05$ vs. sham group, ${ }^{\#} P<0.05$ vs. I/R group.

3.3. Dioscin Scavenges ROS in Perfused H9C2 Cells. Considering the results from the in vivo experiments, we further verified whether dioscin abolishes ROS accumulation in myocytes in an oxidant condition. We further performed dihydroethidium (DHE) staining to detect the level of ROS in $\mathrm{H}_{2} \mathrm{O}_{2}$-incubated $\mathrm{H} 9 \mathrm{C} 2$ cells, showing that dioscin treatment markedly attenuated the elevated levels of ROS production (Figures 5(a) and 5(b)). Because the nicotinamide adenine dinucleotide phosphate (NADPH) oxidase 2 (Nox2) plays a central role in catalyzing the production of superoxide from oxygen, we identified that the expression of Nox2 was obviously increased in $\mathrm{H}_{2} \mathrm{O}_{2}$-cultured $\mathrm{H} 9 \mathrm{C} 2$, and the increase was prevented after dioscin treatment (Figure 5(c)). These results verified that dioscin alleviates perfused injury via downregulation of the oxidant response.

\section{Discussion}

In this study, we demonstrated that dioscin, as a natural product, showed a cardioprotective role in response to myocardial ischemic/reperfusion (MI/R) injury. Dioscin has a therapeutic effect via downregulation of oxidant stress, reflecting from the elevated levels of the antioxidant enzyme activities, accompanying with the ROS scavenger. These results exhibited that dioscin has potent effects for the treatment of MI/R injury.

Multiple events take part in the pathogenesis of $M I / R$, including accumulation of ROS, inflammation, perturbation of calcium handing, and metabolic derangements. Considering ROS as the primary cause among these stimuli, the pharmacological antagonists of accumulated succinate sufficiently ameliorated in vivo myocardial ischemia/reperfusion injury 


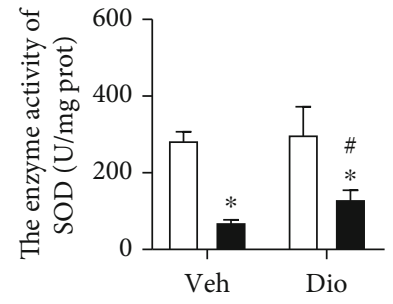

$\square$ Sham

D/R

(a)

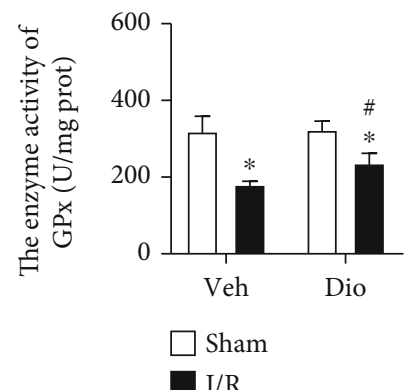

(c)

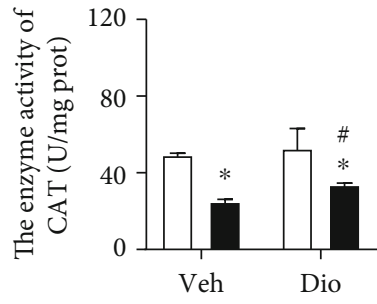

$\square$ Sham

I/R

(b)

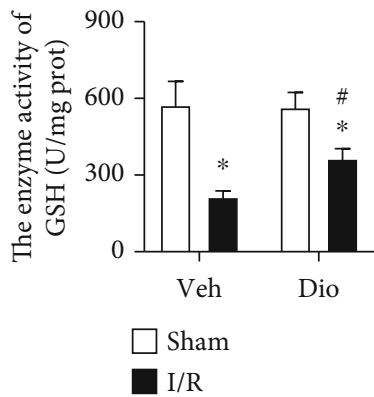

(d)

Figure 4: Dioscin modulates antioxidant status in IR hearts. The enzyme activities for (a) SOD, (b) CAT, (c) GPx, and (d) GSH. $n=6$ each group, repeated twice. Data are mean \pm SD. ${ }^{*} P<0.05$ vs. sham group, ${ }^{\#} P<0.05$ vs. I/R group.

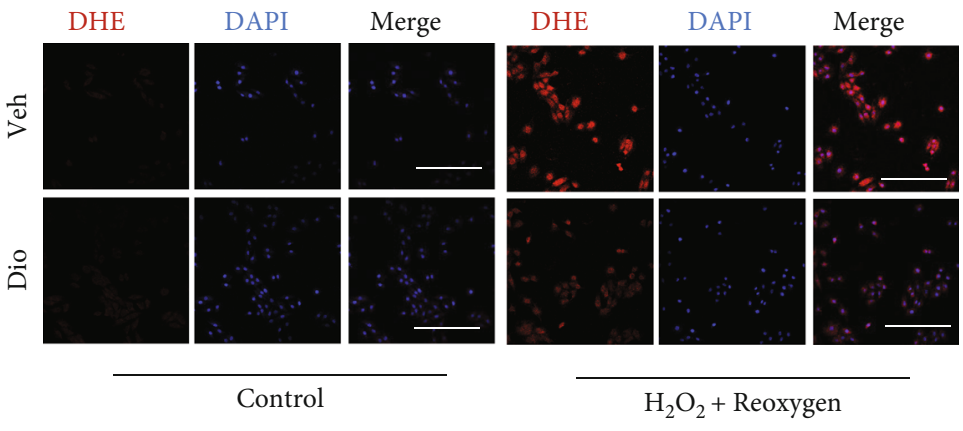

(a)

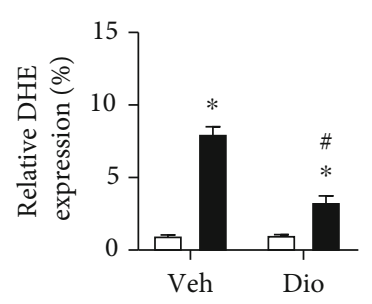

$\square$ Control

$\mathrm{H}_{2} \mathrm{O}_{2}+$ Reoxygen

(b)

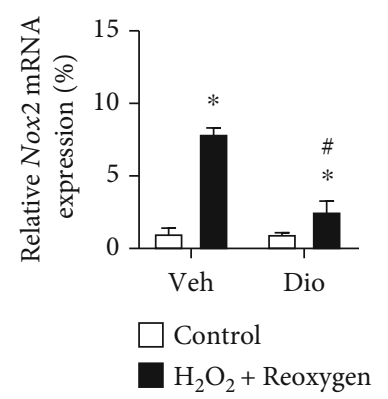

(c)

FIGURE 5: Dioscin abolishes the accumulation of ROS production in H9C2 cells. Representative images of double staining of H9C2 cells with dihydroethidium (DHE) (red) and DAPI (blue) $($ bar $=50 \mu \mathrm{m}), n=3$ each group. (b) The quantification of relative expression of reactive oxygen species. (c) The mRNA expression of Nox2. $n=6$ each group. Data are mean \pm SD. ${ }^{*} P<0.05$ vs. control group, ${ }^{\#} P<0.05$ vs. $\mathrm{H}_{2} \mathrm{O}_{2}+$ reoxygen group. 
via repressing extensive ROS generation [29]. Further, uric acid aggravates MI/R-induced activation of the NOD-like receptor pyrin domain-containing protein 3 (NLRP3) inflammatory cascade and pyroptosis by promoting ROS generation, while inflammasome inhibitors and ROS scavengers partly reverse the injury [30]. What is more, ROS scavenger Nacetyl cysteine (NAC) was able to reduce the amount of ROS and prevent cell death [31]. Herein, we identified that dioscin was considered as the ROS scavenger in the process of MI/R injury.

Dioscin plays a beneficial role in cardiac protection via reducing the production of ROS. MI/R injury is mediated by the elevated production of ROS, which occurs particularly at reperfusion [32]. The nicotinamide adenine dinucleotide phosphate (NADPH) oxidase family is considered as one major source of ROS in cardiomyocytes [33]. Meanwhile, many antioxidant enzymes participate in the elimination of ROS in response to MI/R injury [34]. Additionally, it was reported that dioscin alleviated doxorubicin-induced cardiotoxicity through modulating miR-140-5p-mediated myocardial oxidative stress [35]. Furthermore, dioscin has renoprotective effects against fructose-induced renal damage via adjusting sirtuin 3mediated oxidative stress [36]. And dioscin significantly protected against renal damage by decreasing blood urea nitrogen and creatinine levels and reversing oxidative stress [37].

Summarily, dioscin ameliorates myocardial ischemia/reperfusion injury through suppressing reactive oxygen species via downregulation of Nox2 and upregulation of the antioxidative enzyme, including SOD, CAT, GPx, and GSH, leading to alleviate cardiac dysfunction. Our results indicated that dioscin, providing a potential therapeutic strategy, would be beneficial for myocardial ischemic/reperfusion injury.

\section{Data Availability}

The data used to support the findings of this study are available from the corresponding authors upon request.

\section{Conflicts of Interest}

The authors declare that they have no conflicts of interest.

\section{Authors' Contributions}

Qiulun Lu and Pingxi Xiao designed the study and revised the manuscript. Dayin Lyu, Qing Tian, and Huitao Qian carried out the experiments, and Dayin Lyu wrote the draft manuscript. Chang He, Tianyu Shen, and Jinxing Xi consulted the corresponding literature for preparation. All authors read and approved the final manuscript. Dayin Lyu and Qing Tian contributed equally to this work.

\section{Acknowledgments}

This study was supported by the grants from the National Natural Science Foundation of China (81970414 to Q.L.) and the Natural Science Foundation of the Jiangsu Higher Education Institutions of China (19KJA35000 to Q.L.).

\section{References}

[1] H. Y. Chen, Z. Z. Xiao, X. Ling, R. N. Xu, P. Zhu, and S. Y. Zheng, "ELAVL1 is transcriptionally activated by FOXC1 and promotes ferroptosis in myocardial ischemia/reperfusion injury by regulating autophagy," $\mathrm{Mol} \mathrm{Med,} \mathrm{vol.} \mathrm{27,} \mathrm{no.} \mathrm{1,}$ p. 14, 2021.

[2] N. Schanze, C. Bode, and D. Duerschmied, "Platelet contributions to myocardial ischemia/reperfusion injury," Front Immunol, vol. 10, p. 1260, 2019.

[3] S. S. Virani, A. Alonso, E. J. Benjamin et al., "Heart disease and stroke statistics-2020 update: a report from the American Heart Association," Circulation, vol. 141, no. 9, pp. e139e596, 2020.

[4] M. ten Hove, C. A. Lygate, A. Fischer et al., "Reduced inotropic reserve and increased susceptibility to cardiac ischemia/reperfusion injury in phosphocreatine-deficient guanidinoacetateN-methyltransferase-knockout mice," Circulation, vol. 111, no. 19, pp. 2477-2485, 2005.

[5] M. I. Ashraf, M. Ebner, C. Wallner et al., “A p38MAPK/MK2 signaling pathway leading to redox stress, cell death and ischemia/reperfusion injury," Cell Commun Signal, vol. 12, no. 1, p. 6, 2014.

[6] S. Hernandez-Resendiz, K. Chinda, S. B. Ong, H. CabreraFuentes, C. Zazueta, and D. . Hausenloy, "The role of redox dysregulation in the inflammatory response to acute myocardial ischaemia-reperfusion injury-adding fuel to the fire," Curr Med Chem, vol. 25, no. 11, pp. 1275-1293, 2018.

[7] H. Zhou, J. Wang, P. Zhu, S. Hu, and J. Ren, "Ripk3 regulates cardiac microvascular reperfusion injury: The role of IP3Rdependent calcium overload, $\mathrm{XO}$-mediated oxidative stress and F-action/filopodia-based cellular migration," Cell Signal, vol. 45, pp. 12-22, 2018.

[8] M. Yang, B. S. Linn, Y. Zhang, and J. Ren, "Mitophagy and mitochondrial integrity in cardiac ischemia-reperfusion injury," Biochim Biophys Acta Mol Basis Dis, vol. 1865, no. 9, pp. 2293-2302, 2019.

[9] K. Raedschelders, D. M. Ansley, and D. D. Chen, "The cellular and molecular origin of reactive oxygen species generation during myocardial ischemia and reperfusion," Pharmacol Ther, vol. 133, no. 2, pp. 230-255, 2012.

[10] A. van der Pol, W. H. van Gilst, A. A. Voors, and P. van der Meer, "Treating oxidative stress in heart failure: past, present and future," Eur J Heart Fail, vol. 21, no. 4, pp. 425-435, 2019.

[11] J. Farías, V. M. Molina, R. A. Carrasco et al., "Antioxidant therapeutic strategies for cardiovascular conditions associated with oxidative stress," Nutrients, vol. 9, no. 9, p. 966, 2017.

[12] S. J. Forrester, D. S. Kikuchi, M. S. Hernandes, Q. Xu, and K. K. Griendling, "Reactive oxygen species in metabolic and inflammatory signaling," Circ Res, vol. 122, no. 6, pp. 877902, 2018.

[13] J. I. Goldhaber and J. N. Weiss, "Oxygen free radicals and cardiac reperfusion abnormalities," Hypertension, vol. 20, no. 1, pp. 118-127, 1992.

[14] R. Rodrigo, "Prevention of postoperative atrial fibrillation: novel and safe strategy based on the modulation of the antioxidant system," Front Physiol, vol. 3, p. 93, 2012.

[15] S. Bae, M. Park, C. Kang et al., "Hydrogen peroxide-responsive nanoparticle reduces myocardial ischemia/reperfusion injury," J Am Heart Assoc, vol. 5, no. 11, 2016. 
[16] F. Tullio, C. Angotti, M. G. Perrelli, C. Penna, and P. Pagliaro, "Redox balance and cardioprotection," Basic Res Cardiol, vol. 108, no. 6, p. 392, 2013.

[17] B. Dong, C. Liu, R. Xue et al., "Fisetin inhibits cardiac hypertrophy by suppressing oxidative stress," J Nutr The Biochemist, vol. 62, pp. 221-229, 2018.

[18] J. du, W. He, C. Zhang et al., "Pentamethylquercetin Attenuates Cardiac Remodeling via Activation of the Sestrins/Keap1/Nrf2 Pathway in MSG-Induced Obese Mice," Biomed Res Int, vol. 2020, article 3243906, pp. 1-10, 2020.

[19] H. H. Liao, N. Zhang, Y. Y. Meng et al., "Myricetin alleviates pathological cardiac hypertrophy via TRAF6/TAK1/MAPK and Nrf 2 signaling pathway," Oxid Med Cell Longev, vol. 2019, p. 6304058, 2019.

[20] Y. Zhang, Y. Cui, S. Dai et al., "Isorhynchophylline enhances Nrf 2 and inhibits MAPK pathway in cardiac hypertrophy," Naunyn Schmiedebergs Arch Pharmacol, vol. 393, no. 2, pp. 203-212, 2020.

[21] L. Zhao, X. Tao, Y. Qi, L. Xu, L. Yin, and J. Peng, "Protective effect of dioscin against doxorubicin-induced cardiotoxicity via adjusting microRNA-140-5p-mediated myocardial oxidative stress," Redox Biol, vol. 16, pp. 189-198, 2018.

[22] C. Kong, D. Lyu, C. He, R. Li, and Q. Lu, "DioscinelevateslncRNA MANTIS in therapeutic angiogenesis for heart diseases," Aging Cell, vol. 20, no. 7, p. e13392, 2021.

[23] X. Tao, X. Wan, Y. Xu et al., "Dioscin attenuates hepatic ischemia-reperfusion injury in rats through inhibition of oxidative-nitrative stress, inflammation and apoptosis," Transplantation, vol. 98, no. 6, pp. 604-611, 2014.

[24] L. Zheng, X. Han, Y. Hu et al., "Dioscin ameliorates intestinal ischemia/reperfusion injury via adjusting miR-3515p/MAPK13-mediated inflammation and apoptosis," Pharmacol Res, vol. 139, pp. 431-439, 2019.

[25] Y. Hu, X. Tao, X. Han et al., "Dioscin attenuates gastric ischemia/reperfusion injury through the down- regulation of $\mathrm{PKC} / \mathrm{ERK} 1 / 2$ signaling via $\mathrm{PKC}_{\alpha}$ and $\mathrm{PKC}_{\beta 2}$ inhibition," Chem Biol Interact, vol. 258, pp. 234-244, 2016.

[26] E. Gao, Y. H. Lei, X. Shang et al., "A novel and efficient model of coronary artery ligation and myocardial infarction in the mouse," Circ Res, vol. 107, no. 12, pp. 1445-1453, 2010.

[27] J. Chen, Z. P. Huang, H. Y. Seok et al., "mir-17-92 cluster is required for and sufficient to induce cardiomyocyte proliferation in postnatal and adult hearts," Circ Res, vol. 112, no. 12, pp. 1557-1566, 2013.

[28] M. Zhang, J. Lin, S. Wang et al., "Melatonin protects against diabetic cardiomyopathy through Mst1/Sirt3 signaling," $J$ Pineal Res, vol. 63, no. 2, 2017.

[29] E. T. Chouchani, V. R. Pell, E. Gaude et al., "Ischaemic accumulation of succinate controls reperfusion injury through mitochondrial ROS," Nature, vol. 515, no. 7527, pp. 431-435, 2014.

[30] S. Shen, F. He, C. Cheng, B. L. Xu, and J. L. Sheng, "Uric acid aggravates myocardial ischemia-reperfusion injury _via_ ROS/NLRP3 pyroptosis pathway," Biomed Pharmacother, vol. 133, p. 110990, 2021.

[31] C. Beltran, R. Pardo, D. Bou-Teen et al., "Enhancing glycolysis protects against ischemia-reperfusion injury by reducing ROS production," Metabolites, vol. 10, no. 4, p. 132, 2020.

[32] S. Cadenas, "ROS and redox signaling in myocardial ischemiareperfusion injury and cardioprotection," Free Radic Biol Med, vol. 117, pp. 76-89, 2018.
[33] K. Bedard and K. H. Krause, "The NOX family of ROSgenerating NADPH oxidases: physiology and pathophysiology," Physiol Rev, vol. 87, no. 1, pp. 245-313, 2007.

[34] J. M. Yoo, B. D. Lee, D. E. Sok, J. Y. Ma, and M. R. Kim, "Neuroprotective action of $\mathrm{N}$-acetyl serotonin in oxidative stress-induced apoptosis through the activation of both TrkB/CREB/BDNF pathway and Akt/Nrf2/Antioxidant enzyme in neuronal cells," Redox Biol, vol. 11, pp. 592599, 2017.

[35] L. Zhao, X. Tao, Y. Qi, L. Xu, L. Yin, and J. Peng, "Corrigendum to 'Protective effect of dioscin against doxorubicininduced cardiotoxicity via adjusting microRNA-140-5p-mediated myocardial oxidative stress' [Redox Biol.], 2018, 16: 189198," Redox Biol, vol. 26, p. 101303, 2019.

[36] Y. Qiao, L. Xu, X. Tao et al., "Protective effects of dioscin against fructose-induced renal damage via adjusting Sirt3mediated oxidative stress, fibrosis, lipid metabolism and inflammation," Toxicol Lett, vol. 284, pp. 37-45, 2018.

[37] M. Qi, L. Yin, L. Xu et al., "Dioscin alleviates lipopolysaccharide-induced inflammatory kidney injury _via_ the microRNA let-7i/TLR4/MyD88 signaling pathway," Pharmacol Res, vol. 111, pp. 509-522, 2016. 